\title{
ANÁLISIS MULTIESCALA DE CICLICIDADES EN REGISTROS DE POZO A PARTIR DE LA TRANSFORMADA DE ONDÍCULA, APLICADO AL CAMPO COLORADO
}

\author{
Julie Bedoya ${ }^{1}$; Ricardo Mier ${ }^{1}$; Luis Cruz ${ }^{1}$
}

DOI: http://dx.doi.org/10.18273/revbol.v38n2-2016007 (c) (1) అ

Forma de citar: Bedoya, J., Mier, R., y Cruz, L. 2016. Análisis multiescala de ciclicidades en registros de pozo a partir de la transformada de ondícula, aplicado al campo Colorado. Boletín de Geología, 38(2): 101-108.

\section{RESUMEN}

Este artículo muestra la aplicación de la herramienta matemática de la transformada de ondícula para el análisis de registros de Rayos Gamma (GR) y Resistivos (ILD), en los pozos C54, C56, C63, C65, C66 y C68 del campo Colorado, ubicado en la Cuenca del Valle Medio del Magdalena (Colombia). Los registros fueron procesados a través de la herramienta de la transformada de ondícula en el Wavelet Toolbox de MATLAB 7.1. Con esta herramienta se obtuvieron 12 escalogramas que permitieron corroborar los topes estratigráficos de las (Zonas B1-B2) y la Zona C de la Formación Mugrosa. Los topes fueron establecidos por la presencia de señales de alta intensidad en los escalogramas. Con la transformada de ondícula discreta se realizó un análisis multi-resolución de registros de Rayos Gamma (GR) en la Zona B de la Formación Mugrosa para los pozos C63, C65, C66 y C68. En los pozos C63, C65 y C68 se encontró que la relación 1:2 muestra las periodicidades de precesión 20 ka y oblicuidad $41 \mathrm{ka}$ de los ciclos de Milankovitch. Sin embargo, la ausencia de una descripción sedimentológica detallada no permite mostrar la relación de estas ciclicidades con el modelo sedimentario para cada pozo. Estas periodicidades se relacionaron con las electroformas de canal y llanura de inundación definidas en el modelo sedimentológico y estratigráfico de Campo Colorado.

Palabras clave: transformada de ondícula, escalogramas, topes estratigráficos, análisis multi-resolución, Formación Mugrosa.

\section{MULTISCALE ANALYSIS OF CYCLICITIES IN WELL LOGS FROM THE WAVELET TRANSFORM, APPLIED TO CAMPO COLORADO}

\begin{abstract}
This paper shows the application of the mathematical tool wavelet transform to the analysis of Gamma Ray (GR) and Resistivity (ILD) logs from the C54, C56, C63, C65, C66 and C68 wells of campo Colorado, located in the Middle Magdalena Basin (Colombia). Records were processed through the tool wavelet transform in the Wavelet Toolbox from MATLAB 7.1. With this tool, 12 scalograms were obtained, which allowed corroborating stratigraphic tops of the Zones B1-B2 and the Zone C of the Mugrosa Formation. Tops were established by the presence of high intensity signals in scalograms. With the discrete wavelet transform the multiresolution analysis of logs GR was realized in the Zone B of the Mugrosa Formation from C63, C65, C66 and C68 wells. In the wells C63, C65 and C68 it was found that the relation 1:2 showed the periodicities of $20 \mathrm{ka}$ precessions and $41 \mathrm{ka}$ obliquity from Milankovitch cycles. However, the absence of detailed sedimentological description does not let showing the relationship of these cyclicities with the sedimentary model for each well. These periodicities are related with electroforms channel and flood plain defined sedimentological and stratigraphic model of campo Colorado.
\end{abstract}

Keywords: wavelet transform, scalograms, stratigraphic tops, multiresolution analysis, Mugrosa Formation.

${ }^{1}$ Universidad Industrial de Santander, Escuela de Geología, Bucaramanga, Colombia. juliebedoyamontilla@hotmail.com. 


\section{INTRODUCCIÓN}

El campo Colorado se encuentra en la cuenca del Valle Medio del Magdalena, Colombia. (FIGURA 1). La secuencia estratigráfica encontrada en los pozos del Campo Colorado atraviesa de tope a base las Zonas B y $\mathrm{C}$ de la Formación Mugrosa; estos topes fueron definidos por Mier et al. (2013), a partir de la interpretación de las curvas de registros SP tomadas en cada uno de los pozos. En este trabajo se pretende validar estos topes estratigráficos, con una metodología que utiliza la herramienta matemática de la transformada de ondícula, la cual ha sido aplicada desde la década de los ochenta para la resolución de problemas relacionados con señales no estacionarias presentes en las ondas sísmicas y registros de pozo (Montejo y Suarez, 2007).

En general, las investigaciones de la transformada de ondícula han respondido a dos intereses principales, uno enfocado a la aplicación de la transformada de ondícula continua para el reconocimiento de los ciclos de Milankovitch en registros de pozo (Yu et al., 2008) y el otro con la aplicación de la transformada de ondícula discreta.
Las transformadas de ondícula continua y discreta se han aplicado a datos de registros geofísicos de pozos para obtener pseudolongitudes de onda asociadas a periodos de sedimentación o ciclos de Milankovitch (Coconi et al., 2010), y en la definición de características no visibles en registros de pozos, a partir del análisis de escalogramas (Sánchez, 2011).

El énfasis de este estudio fue presentar la metodología que emplea el software de computación matemática MATLAB 7.1, en la aplicación de la transformada de ondícula continua para el análisis de escalogramas en registros de inducción profunda (ILD) y registros de Rayos Gamma (GR), en los pozos C54, C56, C63, C65, C66 y C68 del Campo Colorado, con el objetivo de caracterizar los topes estratigráficos de las zonas B1-B2 y la Zona C de la Formación Mugrosa. La caracterización de la Zona B de la Frm. Mugrosa permitió la realización del análisis multiescala mediante la aplicación de la transformada de ondícula discreta, la cual se basó en la metodología propuesta por Yu et al. (2008) para el análisis de ciclicidades en registros de pozo.

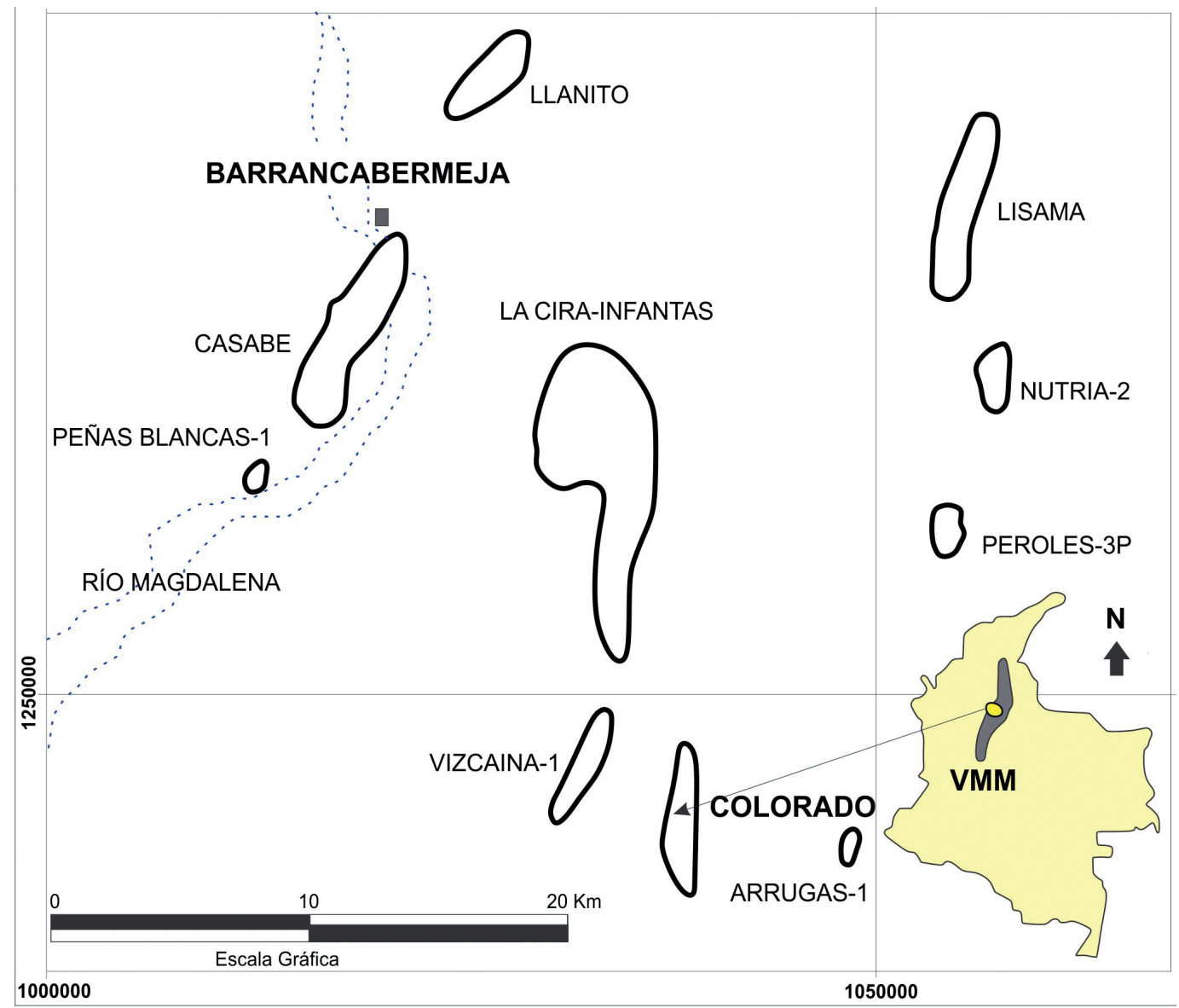

FIGURA 1. Ubicación del campo Colorado en la cuenca del Valle Medio del Magdalena (VMM). Modificado de Ecopetrol (2003). 


\section{MARCO ESTRATIGRÁFICO}

El nombre de la Formación Mugrosa fue propuesto por McGill (1928) en Morales et al. (1958); su nombre fue tomado de la quebrada Mugrosa, donde se encuentra su sección tipo. Su espesor varía entre 500 y 800 m., el contacto inferior de la Formación Mugrosa es discordante con la Formación Esmeraldas y el contacto superior es concordante con la Formación Colorado (FIGURA 2). Su edad es considerada del
Eoceno tardío - Oligoceno temprano (Royero y Clavijo, 2001).

La unidad en su parte inferior está compuesta por areniscas gris verdosas, de grano fino a medio, con intercalaciones de lodolitas grises a azulosas y algunas capas de areniscas conglomeráticas. La parte media consta de lutitas moteadas con algunas intercalaciones de arenisca. Hacia la parte superior hay lodolitas moteadas, fosilíferas (Royero y Clavijo, 2001).

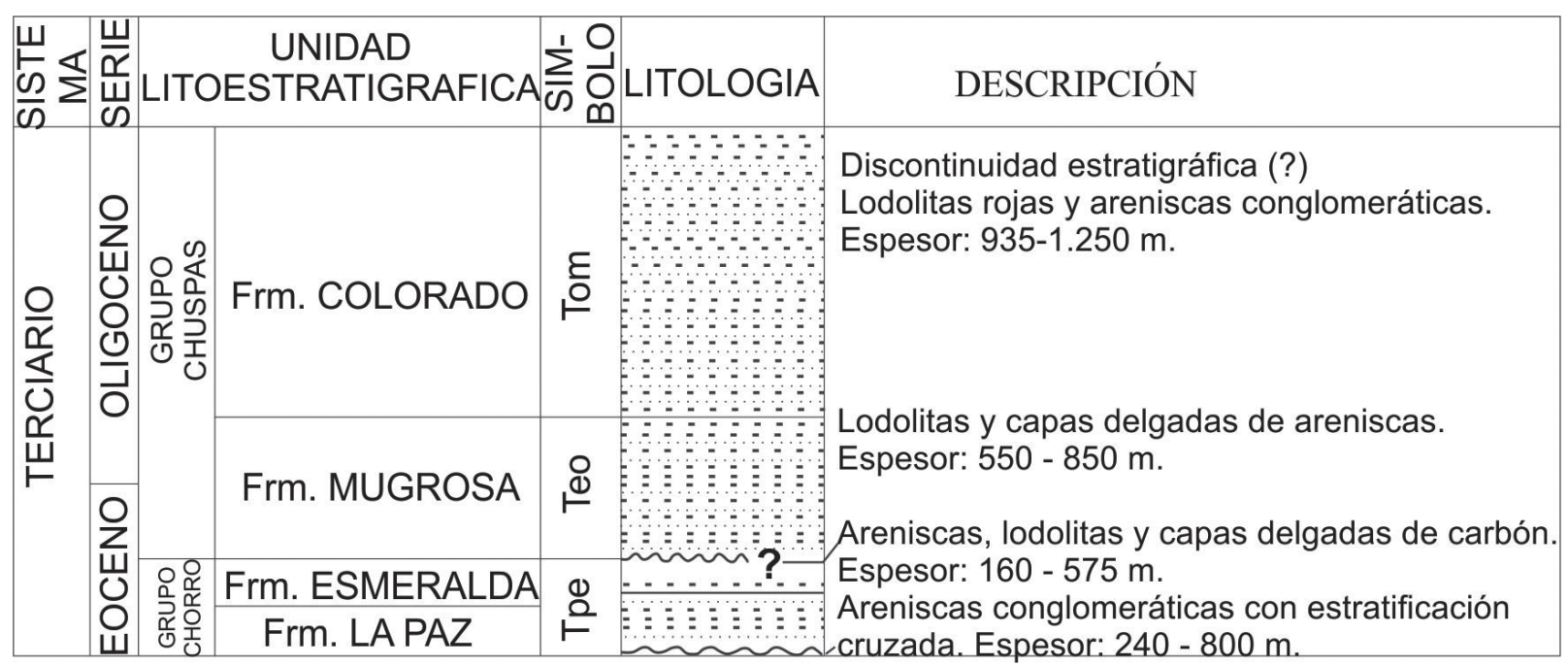

FIGURA 2. Estratigrafía del terciario en el Valle Medio del Magdalena. Modificado de Royero y Clavijo (2001).

La Formación Mugrosa en el campo Colorado suprayace el "Horizonte Fosilífero de los Corros" y en su parte superior se presenta el "Horizonte Fosilífero de Mugrosa" (Royero y Clavijo, 2001). Esta formación se ha divido en dos zonas; la parte superior o Zona B y la parte inferior o Zona $\mathrm{C}$, separadas por intervalos potentes de lodolitas. La Zona B, está compuesta por lodolitas grises-verdosas y en algunos casos varicoloreadas, con un espesor de $20 \mathrm{~m}$. Presenta algunos intervalos de areniscas, de grano fino a medio, subangualares a subredondeadas, con espesores máximos de $1,5 \mathrm{~m}$. La Zona $\mathrm{C}$ está compuesta por sucesiones granodecrecientes de areniscas feldespáticas, de grano medio a grueso, angulares, de regular calibrado, con un espesor promedio de $2 \mathrm{~m}$. En algunas ocasiones presenta sucesiones de gravas, con una extensión lateral que no sobrepasa los 10 $\mathrm{m}$. Las lodolitas de esta zona son limolitas arenosas grises a verdes, con espesores máximos de $15 \mathrm{~cm}$ y se encuentran separando a los cuerpos de areniscas feldespáticas (Fonseca y García, 2009).
La sedimentación de la Formación Mugrosa tuvo lugar en un ambiente de tipo fluvial meandriforme, caracterizado por una marcada migración lateral del cauce, de mediana sinuosidad, con barras alternantes, con presencia de los elementos arquitecturales tipo "crevasse splay" y en menor proporción "crevasse channel", donde el desarrollo de paleosuelos es poco, pero la construcción de depósitos de llanura de inundación es fuerte (Fonseca y García, 2009).

En este estudio, se tomó la subdivisión de la Formación. Mugrosa en las subzonas B1-B2 y C1-C2 propuesta por Ecopetrol (2003), las cuales fueron definidas a partir de la interpretación de los registros SPN (Potencial espontáneo normalizado) y microresistivos.

\section{MARCO TEÓRICO}

La transformada de ondícula es una herramienta matemática, que permite identificar en señales no estacionarias presentes en las ondas sísmicas y en los 
registros de pozo: discontinuidades, cambios abruptos, comienzos y finales de eventos. Su uso principal se basa en el procesamiento de señales en todas las escalas y distintas posiciones. Es decir, las señales son escaladas de una ondícula madre y durante su procesamiento se puede localizar un evento simultáneamente en el dominio temporal (espacio) y frecuencial (escala) (Misiti et al., 2007).

\section{Transformada de ondícula continua}

El procesamiento de la transformada de ondícula continua consiste en comparar la señal con ciertas ondículas obtenidas de una ondícula madre, convirtiendo la señal original en una señal tanto en el dominio del tiempo como en el de frecuencia, representando la señal procesada en gráficos tiempo - frecuencia, denominados escalogramas (Misiti et al., 2007).

\section{Transformada de ondícula discreta}

La transformada de ondícula discreta consiste en descomponer una señal en una cantidad de frecuencias mediante la aplicación de filtros multiniveles denominados multi-resolución, de esta manera, se obtiene información detallada de la señal. Para descomponer la señal se debe seleccionar las ondículas que tengan la propiedad de ortonormalidad, la cual permite realizar una reconstrucción discreta de la señal (Misiti et al., 2007).

\section{METODOLOGÍA}

La metodología empleada fue un proceso de dos etapas que resultó en la definición de topes estratigráficos de las Zonas $\mathrm{B}_{1}-\mathrm{B}_{2}$ y la Zona $\mathrm{C}$ de la Formación Mugrosa. Estas dos etapas consisten en la selección y procesamiento de registros ILD y GR para seis pozos localizados en el sector Norte, Centro y Sur del campo Colorado VMM (FIGURA 3).

En la primera etapa, el criterio de selección de los registros de pozos se basó en descartar aquellos con falta de información y presencia de valores nulos. Debido a eso, solo se seleccionaron los registros que mostraron continuidad en profundidad y alcanzaron el tope de la Frm. Mugrosa, que es la unidad de interés de este estudio.

En la segunda etapa, la metodología usada en el procesamiento de la transformada de ondícula continua tiene como base la elaboración de escalogramas a partir de registros ILD y GR; para esta etapa, se generaron 12 escalogramas utilizando escalas altas, que permitan cumplir con los objetivos propuestos: definir $\mathrm{y}$ corroborar topes estratigráficos de las $\left(\right.$ zonas $\left.\mathrm{B}_{1}-\mathrm{B}_{2}\right)$ de la Frm. Mugrosa, en los pozos C63, C65, C66 y C68 ubicados en el sector Centro y Sur del campo Colorado (FIGURA 3) y determinar la existencia de los topes estratigráficos de las zonas C1-C2 de la Frm. Mugrosa, en los pozos C54 y C56 ubicados en el sector Norte del campo Colorado (FIGURA 3).

Con los topes estratigráficos de la Zona B de la Frm. Mugrosa se continuó con el procesamiento de la transformada de ondícula discreta a partir de registros GR, se pasó primero por la elaboración de cuatro escalogramas utilizando escalas bajas, que permitan realizar el análisis multi-resolución en los pozos C63, C65, C66 y C68. Para este análisis, se utilizó la metodología propuesta por $\mathrm{Yu}$ et al. (2008) para el reconocimiento de los ciclos de Milankovitch en registros de pozo.

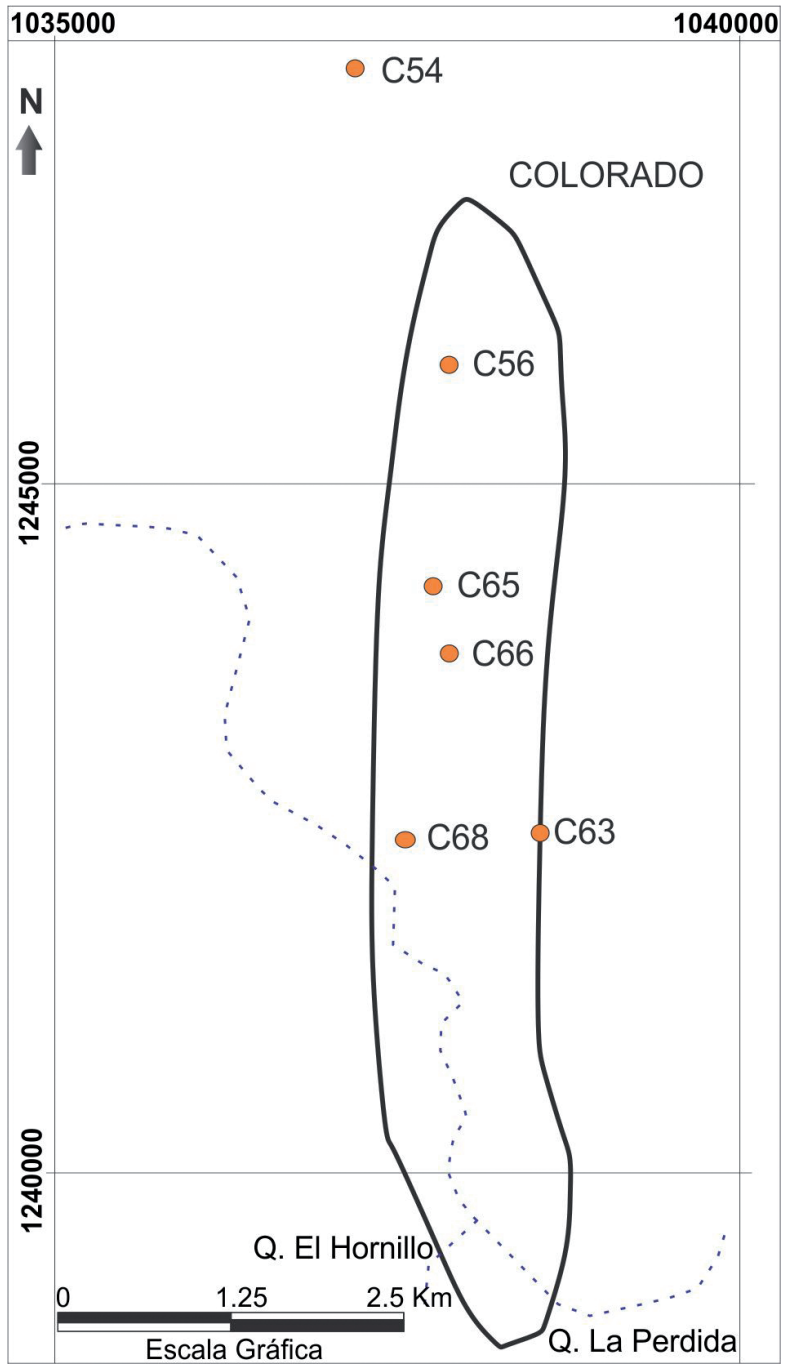

FIGURA 3. Mapa mostrando la localización de los pozos usados en el estudio. 


\section{RESULTADOS Y DISCUSIONES}

En el presente trabajo se utilizaron dos trasformadas de ondícula para analizar los registros ILD y GR en los pozos C54, C56, C63, C65, C66 y C68 en Campo Colorado. La primera: la transformada de ondícula continua, se caracteriza por permitir la elaboración de escalogramas con los que se pudieron definir los topes estratigráficos de la Formación Mugrosa. La segunda: La transformada de ondícula discreta, se caracteriza por permitir el análisis multi-resolución con el que se pudo definir la presencia de ciclicidades en los pozos C63, C65, C66 y C68.

\section{Análisis de escalogramas}

En el análisis de los registros ILD y GR mediante la aplicación de la transformada de ondícula continua, se generaron escalogramas que fueron elaborados en el software MATLAB 7.1, mediante la opción Continuous Wavelet 1-D del módulo Wavelet Toolbox. Para cada uno de los escalogramas se realizaron pruebas con las ondículas Symlet, Daubechies y Coiflet, y se encontró que la ondícula de Coiflet 3 permite observar con mayor claridad la presencia de señales de alta intensidad.

Además, se realizaron pruebas para definir los rangos de escalas altas y escalas bajas para procesar los escalogramas. En este caso específico se define el término escalas altas a rangos con un valor máximo de 1.400 y un valor mínimo de 1; por esta razón son utilizadas con el interés de tener una visión general del comportamiento de los registros a analizar. De esta forma se elaboró un total de ocho escalogramas para definir y corroborar topes estratigráficos de las zonas B1- B2 de la Formación Mugrosa, en los pozos C63, C65, C66 y C68.

En estos pozos se logró identificar, para los escalogramas de registros ILD, la presencia de señales de alta intensidad o anomalías localizadas en los valores máximos del rango de escalas altas, mientras que en los escalogramas de los registros GR se observó la presencia de señales de alta intensidad localizadas en los valores medios del rango de escalas. Debido a esto se consideró procesar los registros GR con un rango de escalas bajas, con el propósito de encontrar señales de alta intensidad localizadas en los valores máximos del rango de escalas bajas, que permitan tener una visión detallada del comportamiento del registro GR. De este modo, se logró identificar en los escalogramas de registros GR señales de alta intensidad localizadas en los valores máximos del rango de escalas bajas. Finalmente, en cada uno de los pozos, los escalogramas de registros ILD procesados a escalas altas y los escalogramas de registros GR procesados a escalas bajas, revelaron la presencia de topes estratigráficos localizados en las mismas profundidades en cada registro de pozo, lo que permite demostrar la utilidad de la transformada de ondícula continua en la definición de topes estratigráficos.

Los resultados obtenidos muestran en el pozo C63 (FIGURA 4) los escalogramas de registros ILD y GR para el intervalo de profundidad de $1.820 \mathrm{ft}$ a $3.410 \mathrm{ft}$, donde se visualiza los topes estratigráficos de las zonas B1- B2 de la Formación Mugrosa. El tope de la zona $\mathrm{B} 1$, se definió por la presencia de una anomalía de alta intensidad en el registro ILD a unos $25 \mathrm{ft}$ por encima del tope de la Zona B, que fue definido por Mier et al. (2013). En los dos escalogramas se distinguen, a $2.275 \mathrm{ft}$, una anomalía de alta intensidad que permitió definir la base de la Zona B1 y el tope de la Zona B2. Posteriormente, en el intervalo de profundidad de 2.275 $\mathrm{ft}$ a $3.040 \mathrm{ft}$, se observan unas cortas longitudes de onda que corresponden a las intercalaciones de lodolitas y areniscas como se muestra en la columna estratigráfica (FIGURA 4). En la profundidad de $3.040 \mathrm{ft}$ se presenta una anomalía de alta intensidad con la que se definió la base de la Zona B2, la cual corresponde con la misma profundidad en la que Mier et al. (2013) definieron la base de la Zona B. Los registros GR de este pozo fueron procesados a detalle con un rango de 100 escalas en el análisis multi-resolución (transformada de ondícula discreta).

En la Zona B de los escalogramas de los pozos C66 y C68, se encontró que los topes estratigráficos obtenidos, tanto en el análisis de escalogramas como los interpretados por Mier et al. (2013) en los registros SP de estos pozos, corresponden a las mismas profundidades así como se muestra en la (TABLA 1). Mientras que en los pozos C63 y C65, los topes estratigráficos solo fueron observados hacia la base de la Zona B (TABLA 1).

Luego se elaboró un total de cuatro escalogramas para determinar la existencia de topes estratigráficos de las zonas $\mathrm{C}_{1}-\mathrm{C}_{2}$ de la Formación Mugrosa en los pozos C54 y C56. En la TABLA 2 se muestran el tope y la base de la Zona $\mathrm{C}$, los cuales fueron obtenidos en el análisis de escalogramas y corresponden con los topes interpretados por Mier et al. (2013) en los registros SP para estos pozos. 


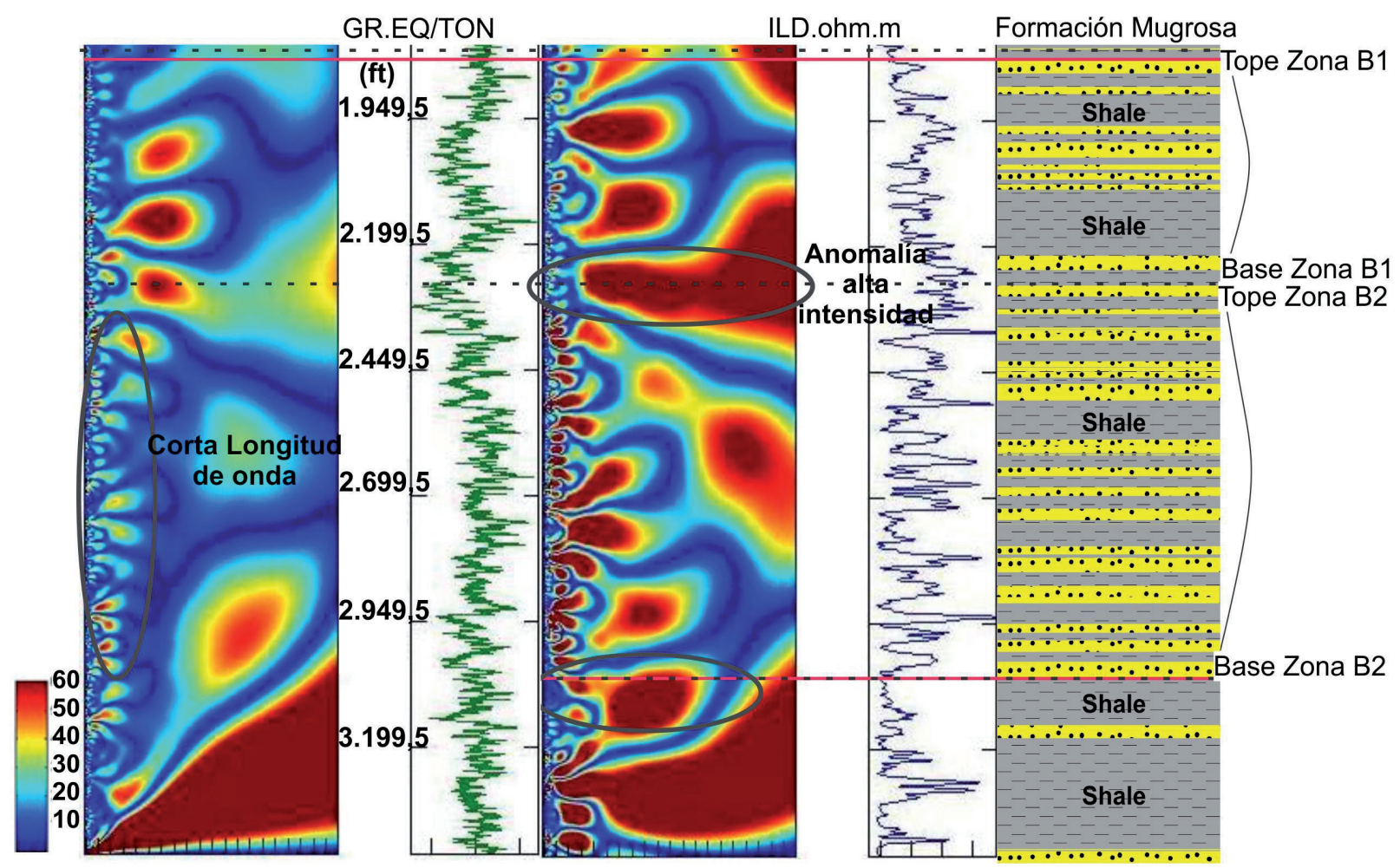

FIGURA 4. Escalogramas de los Registros ILD y GR del pozo C63.

TABLA 1. Topes estratigráficos de las Zonas $\mathrm{B}_{1}-\mathrm{B}_{2}$ de la Formación Mugrosa.

\begin{tabular}{|c|c|c|c|c|c|}
\hline \multirow{2}{*}{ Pozo } & \multirow{2}{*}{$\begin{array}{l}\text { Registros } \\
\text { disponibles }\end{array}$} & \multirow{2}{*}{$\begin{array}{c}\text { Intervalo } \\
\text { procesado } \\
\text { (Pies) }\end{array}$} & \multirow{2}{*}{$\begin{array}{c}\begin{array}{c}\text { Interpretación Registros } \\
\text { SP (Pies) }\end{array} \\
\begin{array}{c}\text { Zona B de la Formación } \\
\text { Mugrosa }\end{array}\end{array}$} & \multicolumn{2}{|c|}{$\begin{array}{c}\text { Escalogramas } \\
\text { Registros (ILD) y (GR), (Pies) }\end{array}$} \\
\hline & & & & Zonas B1-B2 de la & ación Mugrosa \\
\hline \multirow{2}{*}{ C63 } & Resistivos & $700-3.410$ & Tope B: 1.825 & Tope B1: 1.820 & Tope B2: 2.275 \\
\hline & (ILD) y (GR). & $(2.710)$ & Base B: 3.040 & Base B1: 2.275 & Base B2: 3.040 \\
\hline \multirow{2}{*}{ C65 } & Resistivos & $840-3.122$ & Tope B: 1.775 & Tope B1: 1.590 & Tope B2: 2.150 \\
\hline & (ILD) y (GR). & $(2.282)$ & Base B: 2.520 & Base B1: 2.150 & Base B2: 2.520 \\
\hline \multirow{2}{*}{ C66 } & Resistivos & $840-3.360$ & Tope B: 1.825 & Tope B1: 1.825 & Tope B2: 2.151 \\
\hline & (ILD) y (GR). & $(2.520)$ & Base B: 2.475 & Base B1: 2.151 & Base B2: 2.475 \\
\hline \multirow{2}{*}{ C68 } & Resistivos & $700-3.424$ & Tope B: 2.050 & Tope B1: 2.050 & Tope B2: $2.699,5$ \\
\hline & (ILD) y (GR). & $(2.724)$ & Base B: 3.370 & Base B1: $2.699,5$ & Base B2: 3.370 \\
\hline
\end{tabular}

TABLA 2. Topes estratigráficos de las Zonas B y C de la Formación Mugrosa.

\begin{tabular}{|c|c|c|c|c|c|c|}
\hline \multirow[b]{2}{*}{ Pozo } & \multirow[b]{2}{*}{$\begin{array}{c}\text { Registros } \\
\text { disponibles }\end{array}$} & \multirow{2}{*}{$\begin{array}{c}\text { Intervalo } \\
\text { procesado } \\
\text { (Pies) }\end{array}$} & \multicolumn{2}{|c|}{$\begin{array}{c}\text { Interpretación } \\
\text { Registros SP (Pies) }\end{array}$} & \multicolumn{2}{|c|}{$\begin{array}{c}\text { Escalogramas } \\
\text { Registros (ILD) y (GR), (Pies) }\end{array}$} \\
\hline & & & $\begin{array}{l}\text { Zona B de la } \\
\text { Formación } \\
\text { Mugrosa }\end{array}$ & $\begin{array}{c}\text { Zona C de la } \\
\text { Formación } \\
\text { Mugrosa }\end{array}$ & $\begin{array}{c}\text { Zona B de la } \\
\text { Formación } \\
\text { Mugrosa }\end{array}$ & $\begin{array}{c}\text { Zona C de } \\
\text { la Formación } \\
\text { Mugrosa }\end{array}$ \\
\hline \multirow{2}{*}{ C54 } & Resistivos & $2.900-5.880$ & Tope B: 3.425 & Tope C: 5.025 & Tope B1: 3.425 & Tope C: 5.025 \\
\hline & (ILD) y (GR). & $(2.980)$ & Base B: 4.512 & Base C: 5.825 & Base B1: 4.350 & Base C: 5.825 \\
\hline \multirow{2}{*}{ C56 } & Resistivos & $1.500-5.630$ & Tope B: 2.940 & Tope C: 4.550 & Tope B1: 2.850 & Tope C: 4.550 \\
\hline & (ILD) y (GR). & $(4.130)$ & Base B: 3.990 & Base C: 5.550 & Base B1: 3.990 & Base C: 5.550 \\
\hline
\end{tabular}




\section{Análisis multi-resolución}

Una vez definidos los topes estratigráficos de las Zonas B1-B2 de la Formación Mugrosa para los pozos C63, C65, C66 y C68, se diseñó la metodología a utilizar en el procesamiento de la transformada de ondícula discreta a partir de registros GR. Para la elaboración de escalogramas, se seleccionaron en los registros GR los intervalos del tope y la base de las Zonas B1B2 de la Frm. Mugrosa (TABLA1), definidos en el procesamiento de la transformada de ondícula continua. De acuerdo con las pruebas realizadas con anterioridad, a cada registro se le aplicó la ondícula de Coiflet 3 , y en este caso específico, se define el término escalas bajas a rangos con un valor máximo de 100 y un valor mínimo de 1; por esta razón son utilizadas con el interés de tener una visión detallada del comportamiento de los registros que fueron cargados en el software MATLAB 7.1, mediante la opción Continuous Wavelet 1-D del módulo Wavelet Toolbox.

En los escalogramas procesados a escalas bajas se identificaron los valores de las escalas asociadas a las señales de alta intensidad. Con estos valores en el software MATLAB 7.1, mediante la opción Wavelet 1-D del módulo Wavelet Toolbox, se realizó el análisis multi-resolución para los registros GR en los pozos C63, C65, C66 y C68. A partir de estos resultados se empleó la metodología propuesta por Yu et al. (2008) para el análisis de ciclicidades en registros de pozo, la cual consiste en realizar a cada registro GR de pozo el conteo del número de ciclos para cada escala. Posteriormente estos datos se evaluaron con los valores teóricos de la relación 1:2:4.8 de los ciclos de Milankovitch y de esa manera, se definió la relación de los depósitos de la Formación Mugrosa (Zona B) con las periodicidades de excentricidad $100 \mathrm{ka}$, oblicuidad 41 ka y precesión $20 \mathrm{ka}$.

Los resultados obtenidos en el pozo C63, a partir de los escalogramas de registros GR para el intervalo de profundidad del tope de la Zona $\mathrm{B}_{1}$ y la base de la Zona $\mathrm{B}_{2}$, se muestran en la FIGURA 5, donde se pueden observar las señales de alta intensidad que corresponden a las escalas con un valor mínimo a: 16 , un valor medio a: 31 y un valor máximo a: 64. En el análisis multi-resolución se puede observar la descomposición del registro GR en señales de detalle que permiten mostrar para cada una de las escalas las componentes de altas frecuencias. En la Zona $\mathrm{B}_{1}$ para las escalas a: 16 , a: 31 y a: 64 , se encontró un total de 12, 24 y 36 ciclos con una relación 1:2:3, mientras que en la Zona $\mathrm{B}_{2}$, se identificó un total de 19 ,
32 y 64 ciclos con una relación 1:1,7:3,4. De este modo, se logró determinar en el intervalo de la Zona B1 que la relación 1:2:3,2 cumple con los dos primeros valores teóricos de 1:2:4,8 que corresponden a los ciclos orbitales de precesión $20 \mathrm{ka}$ y oblicuidad $41 \mathrm{ka}$, a diferencia del intervalo de la Zona $\mathrm{B}_{2}$, en donde no se encontró ninguna relación con los ciclos de Milankovitch.

Para el intervalo de la Zona $\mathrm{B}_{1}$, en los pozos C63, C65 y C68, los valores de las escalas obtenidas tienen la relación 1: 2, lo que indica la presencia de los ciclos de Milankovitch de precesión y oblicuidad (20 y 41 ka). Estas dos periodicidades permiten identificar la existencia de dos eventos sedimentológicos que se evidencian por la presencia de señales de alta intensidad con un valor mínimo a: 13 y un valor máximo a: 31. Estos eventos se relacionaron tanto con la (1) electroforma de canal, como con (2) la electroforma de llanura de inundación, las cuales fueron definidas por Fonseca y García (2009) a partir de la interpretación de los registros SPN y GR. Sin embargo, la ausencia de una descripción sedimentológica detallada no permite mostrar la relación de estas ciclicidades con el modelo sedimentario para cada pozo.

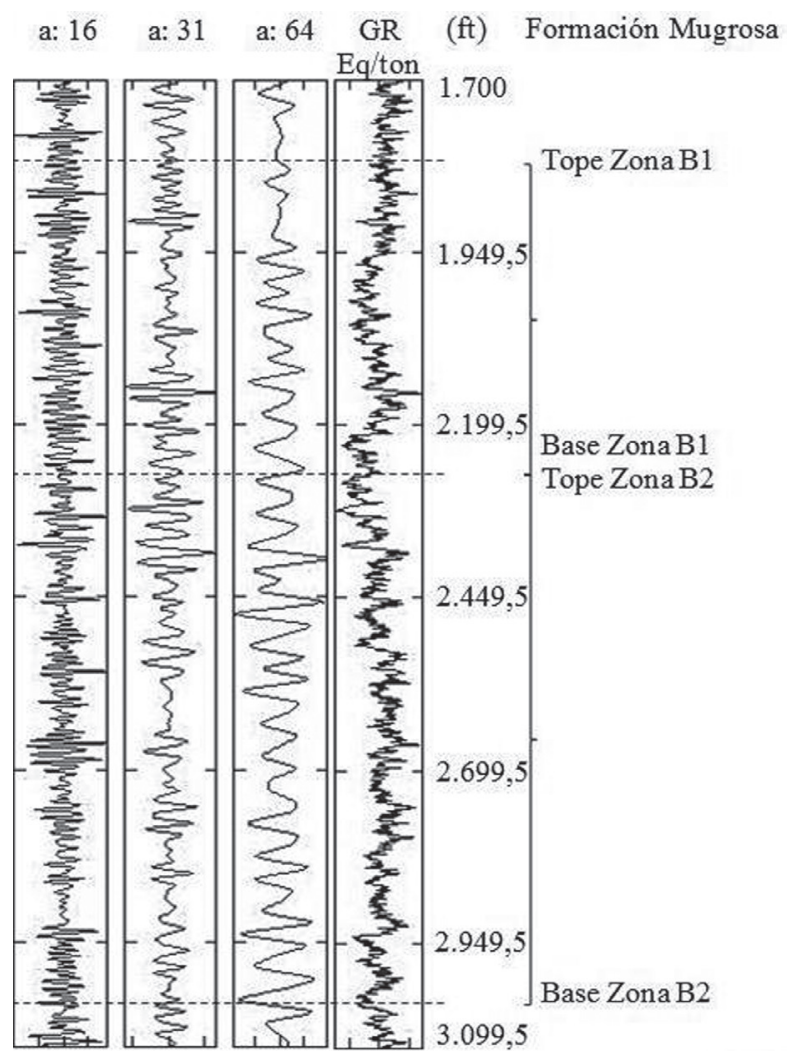

FIGURA 5. Análisis multi-resolución para las escalas (a: 16, a: 31 y a: 64). 
Para el intervalo de la Zona $\mathrm{B}_{2}$, en los pozos C63, C65 y C68 se observó la presencia de señales de alta intensidad con un valor mínimo a: 51 y un valor máximo a: 64 . De este modo, se logró identificar que los valores de las escalas obtenidas no tienen ninguna relación con los ciclos de Milankovitch.

\section{CONCLUSIONES}

El análisis de los registros ILD y GR, para seis pozos en campo Colorado, revela que la aplicación de la transformada de ondícula continua es apropiada para definir topes estratigráficos de las zonas $\mathrm{B}$ y $\mathrm{C}$ de la Formación Mugrosa, y se establece la presencia de señales de alta intensidad como criterio para la definición de topes estratigráficos en los escalogramas.

El análisis de los escalogramas permite localizar señales de alta intensidad, que estudiadas a detalle por el análisis multi-resolución dan como resultado el reconocimiento de periodicidades. Estas periodicidades, se pueden relacionar con la presencia de electroformas para indicar la existencia de eventos sedimentológicos.

Se recomienda emplear la herramienta matemática de la transformada de ondícula en pozos con datos de núcleos de perforación y registros de Rayos Gamma en núcleos, con el fin de identificar la relación entre las periodicidades, las electrofacies y las litofacies, lo que sería una alternativa complementaria para obtener mayor utilidad de la información de pozo.

\section{AGRADECIMIENTOS}

Agradecimientos especiales a Campo Escuela Colorado de la Universidad Industrial de Santander por suministrar los datos para la realización de esta investigación. Adicionalmente, a los geólogos y a los dos evaluadores del Boletín de Geología, que con sus recomendaciones contribuyeron a mejorar este artículo.

\section{REFERENCIAS}

Coconi, E., Ronquillo, G., and Campos, J.O. 2010. Multiscale analysis of well-logging data in petrophysical and stratigraphic correlation. Geofísica Internacional, 49 (2): 55-67.

Ecopetrol. 2003. Diagnóstico y estrategias de recobro Campo Colorado, Gerencia Magdalena Medio, Instituto Colombiano de Petróleo. 109p.

Fonseca, C. y García, J. 2009. Modelo sedimentológico y estratigráfico de la Formación Mugrosa en el Campo Escuela Colorado. Tesis de pregrado, Universidad Industrial de Santander. Bucaramanga.134p.
Mier, R., Chajin, P., Delgado, L., Luna, J., y Palmera, T. 2013. Modelo geológico de Campo Escuela Colorado, Universidad Industrial de Santander, UIS, 143p.

Misiti, M., Misiti, Y., Oppenheim, G., and Poggi, J.M. 2007. Wavelet Toolbox, User's Guide. Versión 4. The Maths Works, Inc.

Montejo, L., y Suarez, L. 2007. Aplicaciones de la transformada ondícula ("wavelet") en Ingeniería estructural. Mecánica Computacional, (26): 2742-2753.

Morales, L., Podesta, D., Hatfield, W.,Tanner, H., Jones, S., Barker, M., O’Donoghue, D., Mohler, C., Dubois, E., Jacobs, C., and Goss, C. 1958. General geology and oil occurrence of the Middle Magdalena Valley, Colombia. In habitat of oil a Symposium American Association of Petroleum Geologists Bulletin, Tulsa. U.S.A, pp. 641-695.

Royero, J.M., y Clavijo, J. 2001. Mapa geológico generalizado del Departamento de Santander. Escala 1:400.000. Memoria explicativa, Bogotá, Colombia: INGEOMINAS.

Sánchez, F. 2011. Análisis multiescala de registros de pozo, Campo Lama; Venezuela. Tesis de pregrado. Universidad Simón Bolívar. Sartenejas. 222p.

Yu, J.F., Sui, F.G., Li, Z.X., Liu, H., and Wang, Y.L. 2008. Recognition of Milankovitch cycles in the stratigraphic record: application of the CWT and the FFT to well-log data. Journal of China University of Mining and Technology, 18(4): 594-598.

Trabajo recibido: abril 9 de 2015

Trabajo aceptado: noviembre 6 de 2015

Manuscrito publicado en internet: noviembre 24 de 2015 